\title{
Supporting Information for First-Principles Study on Transition-Metal- Dichalcogenide/BSe van der Waals Heterostructures: A Promising Water-Splitting Photocatalyst
}

Yi Luo,,$^{\dagger}$ Kai Ren, ${ }^{\dagger}$ Sake Wang, ${ }^{\S}$ Jyh-Pin Chou, ${ }^{\perp}$ Jin $Y u,{ }^{*}{ }^{\dagger}$ Zhengming Sun, ${ }^{\dagger}$ Minglei Sun ${ }^{*,+}$

${ }^{\dagger}$ School of Materials Science and Engineering, Southeast University, Nanjing, Jiangsu 211189, China

${ }^{\ddagger}$ School of Mechanical Engineering, Southeast University, Nanjing, Jiangsu 211189,

China

${ }^{\S}$ College of Science, Jinling Institute of Technology, Nanjing, Jiangsu 211169, China

${ }^{\perp}$ Department of Mechanical Engineering, City University of Hong Kong, Kowloon, Hong Kong SAR 999077, China

\section{Corresponding Authors}

*E-mail: yujin@seu.edu.cn (J. Yu)

*E-mail: mingleisun@outlook.com (M.-L. Sun) 
(a)
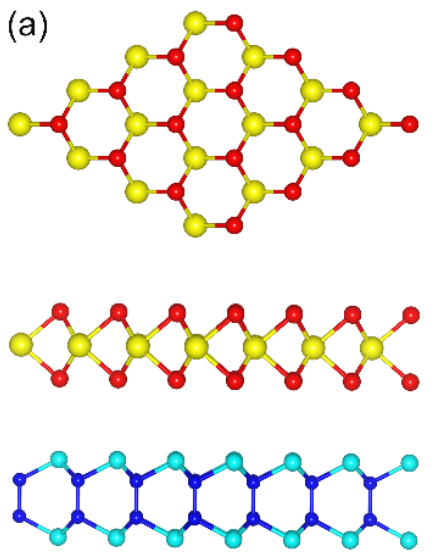

(d)
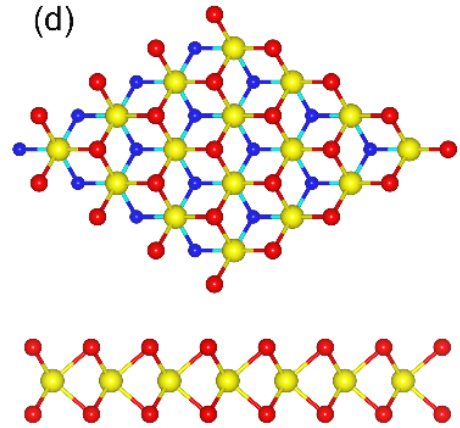

achacho (b)
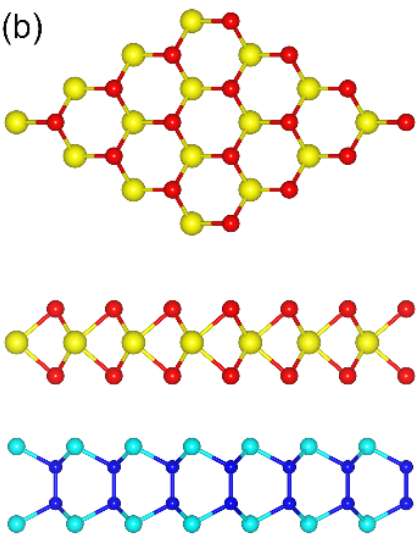

(e)

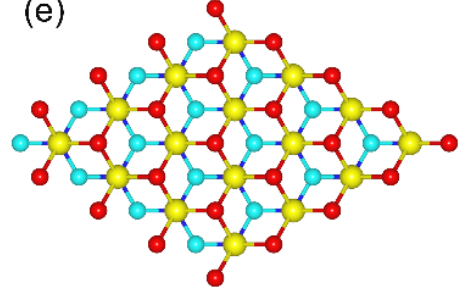

(f)

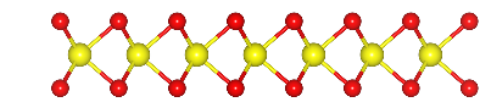

(c)

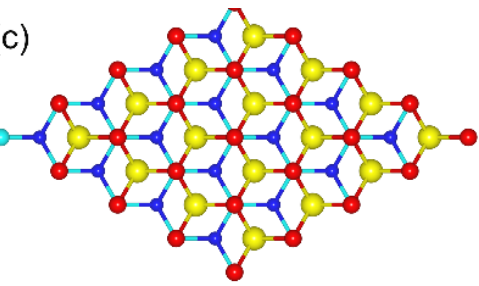

ha

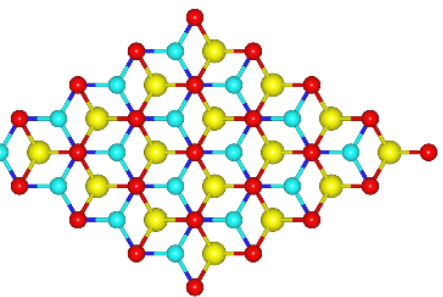

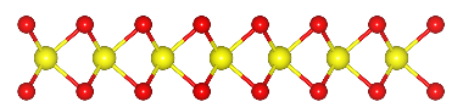

lo

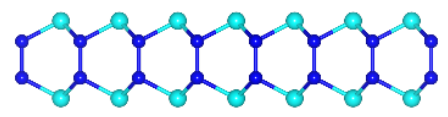

Figure S1. Top and side views of the crystal structures of six stacked $\mathrm{MX}_{2} / \mathrm{BSe}(\mathrm{M}=$ Mo and $\mathrm{W}, \mathrm{X}=\mathrm{S}$ ) heterostructure patterns. The red, yellow, blue, and cyan balls denote $\mathrm{S}, \mathrm{M}(\mathrm{M}=\mathrm{Mo}$ and $\mathrm{W}), \mathrm{B}$, and Se atoms, respectively.

Table S1. The calculated formation energies of all the stacked configurations of TMD/BSe van der Waal heterostructures.

\begin{tabular}{cccccccc}
\hline & & $\mathrm{a}$ & $\mathrm{b}$ & $\mathrm{c}$ & $\mathrm{d}$ & $\mathrm{e}$ & $\mathrm{f}$ \\
\hline$E_{\text {form }}$ & $\mathrm{MoS}_{2} / \mathrm{BSe}$ & -8.09 & -14.34 & -8.32 & -14.10 & -13.06 & -13.64 \\
$\left(\mathrm{meV} / \AA^{2}\right)$ & $\mathrm{WS}_{2} / \mathrm{BSe}$ & -11.03 & -17.47 & -11.15 & -17.36 & -16.09 & -16.78 \\
\hline
\end{tabular}



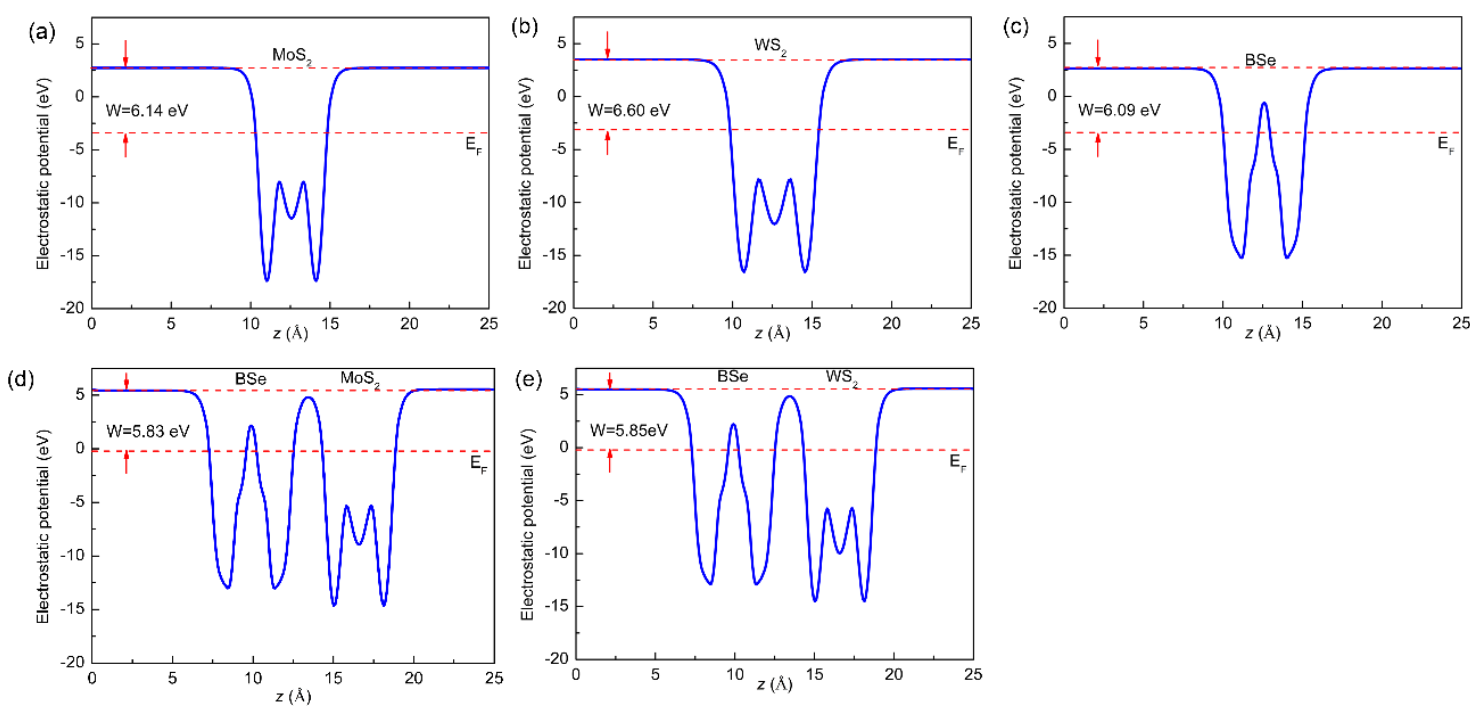

Figure S2. Self-consistent electrostatic potential of (a) pristine $\mathrm{MoS}_{2}$, (b) pristine $\mathrm{WS}_{2}$, (c) pristine $\mathrm{BSe}$, (e) $\mathrm{MoS}_{2} / \mathrm{BSe}$, and (f) $\mathrm{WS}_{2} / \mathrm{BSe}$ heterostructures.

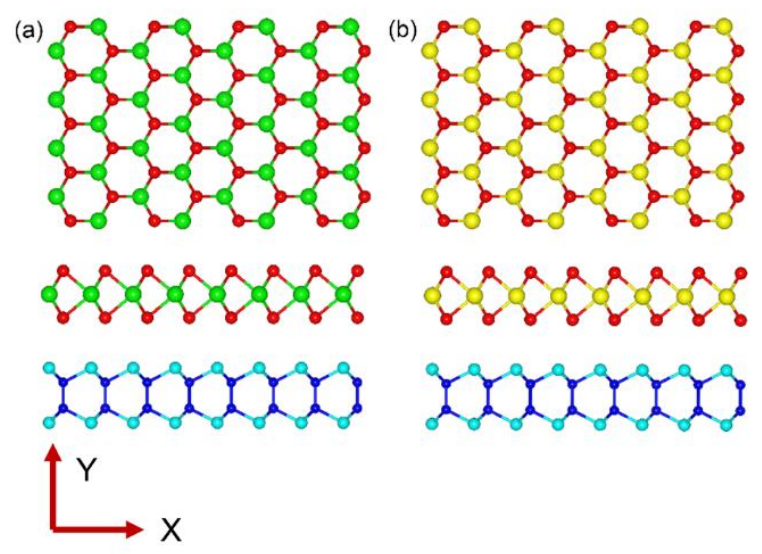

Figure S3. The transport directions of atomic structures for (a) $\mathrm{MoS}_{2} / \mathrm{BSe}$ and (b) $\mathrm{WS}_{2} / \mathrm{BSe}$ heterostructures; the red, yellow, blue, and cyan balls denote $\mathrm{S}, \mathrm{M}(\mathrm{M}=\mathrm{Mo}$ and $\mathrm{W}), \mathrm{B}$, and Se atoms, respectively.
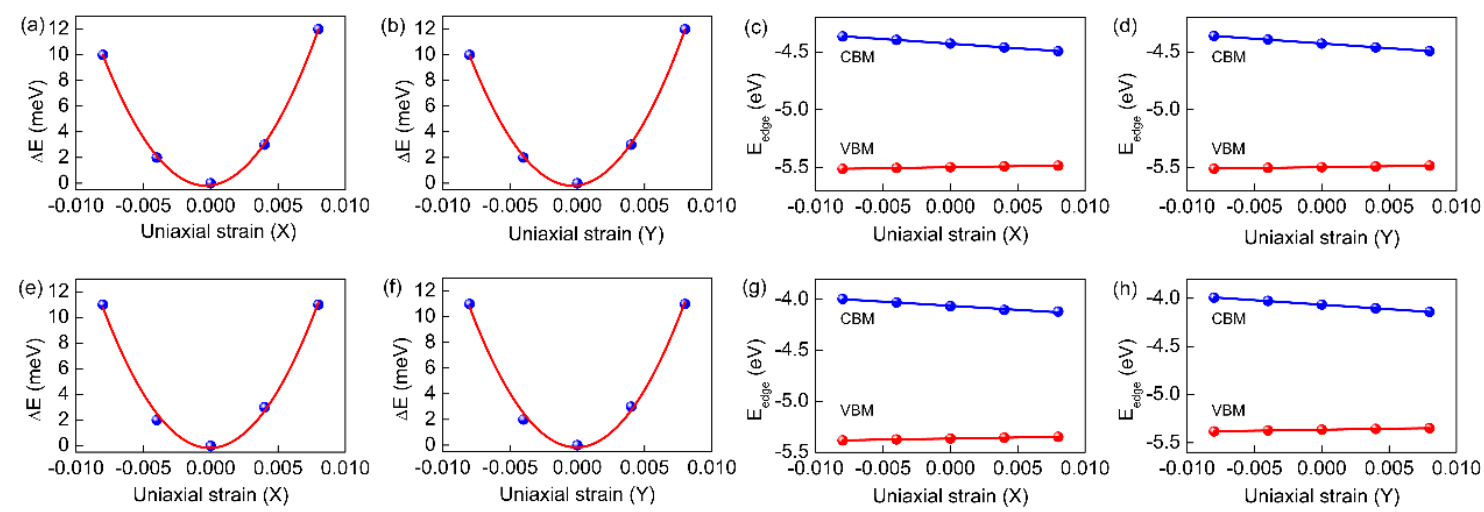

Figure S4. $\Delta \mathrm{E}$ of orthorhombic $\mathrm{MoS}_{2} / \mathrm{BSe}$ heterostructure under uniaxial strain along the (a) $\mathrm{X}$ and (b) $\mathrm{Y}$ directions. Band edge positions of orthorhombic $\mathrm{MoS}_{2} / \mathrm{BSe}$ 
heterostructure under uniaxial strain applied along the (c) $\mathrm{X}$ and (d) $\mathrm{Y}$ directions. $\Delta \mathrm{E}$ of orthorhombic $\mathrm{WS}_{2} / \mathrm{BSe}$ heterostructure under uniaxial strain along the (e) $\mathrm{X}$ and (f) $\mathrm{Y}$ directions. Band edge positions of orthorhombic $\mathrm{WS}_{2} / \mathrm{BSe}$ heterostructure under uniaxial strain applied along the (g) $\mathrm{X}$ and $(\mathrm{h}) \mathrm{Y}$ directions. 